\title{
Is Every Approximate Trajectory of Some Process Near an Exact Trajectory of a Nearby Process?^
}

\author{
Helena E. Nusse ${ }^{1}$ and James A. Yorke ${ }^{2}$ \\ ${ }^{1}$ Rijksuniversiteit Groningen, Faculteit der Economische Wetenschappen, Vakgroep Econometrie, \\ Postbus 800, NL-9700 AV Groningen, The Netherlands \\ ${ }^{2}$ Institute for Physical Science and Technology, and Department of Mathematics, University of \\ Maryland, College Park, MD 20742, USA
}

\begin{abstract}
This paper deals with the problem "Can a noisy orbit be tracked by a real orbit?" In particular, we will study the one-parameter family of tent maps and the one-parameter family of quadratic maps. We write $g_{\mu}$ for either $f_{\mu}$ or $F_{\mu}$ with $f_{\mu}(x)=\mu x$ for $x \leqq \frac{1}{2}$ and $f_{\mu}(x)=\mu(1-x)$ for $x \geqq \frac{1}{2}$, and $F_{\mu}(x)=\mu x(1-x)$. For a given $\mu$ we will say: $g_{\mu}$ permits increased parameter shadowing if for each $\delta_{x}>0$ there exists some $\delta_{\mu}>0$ and some $\delta_{f}>0$ such that every $\delta_{f}$-pseudo $g_{\mu}$-orbit starting in some invariant interval can be $\delta_{x}$-shadowed by a real $g_{\alpha}$-orbit with $\alpha=\mu+\delta_{\mu}$. We show that $g_{\mu}$ typically permits increased parameter shadowing.
\end{abstract}

\section{Contents}

0. Introduction . . . . . . . . . . . . . . . . . . . 364

1. Statement of the Results . . . . . . . . . . . . . . . 365

1-A Noisy Tent Map . . . . . . . . . . . . . . . . 365

1-B Noisy Quadratic Map . . . . . . . . . . . . . . 366

2. Preliminaries . . . . . . . . . . . . . . . . . . 368

2-A Kneading Theory. . . . . . . . . . . . . . . . . . 368

2-B Noisy Cylinders . . . . . . . . . . . . . . . . 369

3. Tent Maps Under the Influence of Noise . . . . . . . . . 370

3-A Tracks of Noisy Orbits. . . . . . . . . . . . . . 370

3-B Noisy Attractor . . . . . . . . . . . . . . . . 371

3-C Length of a Noisy Cylinder . . . . . . . . . . . . 371

3-D Cylinders, Itineraries and Monotonicity . . . . . . . . 372

3-E Pseudo-orbits and Kneading Sequence . . . . . . . . 372

3-F Proofs of the Results . . . . . . . . . . . . . . 373

* Research supported in part by the Netherlands organization for the advancement of pure research (Z.W.O.) and under grant AFOSR-81-0217 
4. Quadratic Maps with Noise. . . . . . . . . . . . . . 374

4-A Noisy Attractor . . . . . . . . . . . . . . . . 374

4-B Noisy Periodic Attractors and Shadowing . . . . . . . 374

4-C Obstruction for Shadowing . . . . . . . . . . . . . 375

4-D When There are only Repelling Periodic Points . . . . . 376

4-E Proofs of the Results . . . . . . . . . . . . . . 377

5. Discussion about more General Results. . . . . . . . . . 378

References . . . . . . . . . . . . . . . . . . . . 378

\section{Introduction}

Studying simple dynamical processes by computer simulation, the question arises whether a trajectory of the numerical process actually corresponds to a trajectory of the real process. We can model these questions by discrete processes, where the Real Process is

$$
y_{n+1}=f\left(y_{n}\right) \text {. }
$$

The Numerical Process can be represented by the same process plus small perturbations (the perturbations represent round off error)

$$
x_{n+1}=f\left(x_{n}\right)+p_{n} \text { with }\left|p_{n}\right| \leqq \delta_{f} \text { for given } \delta_{f}>0 .
$$

For each point $x$ the orbit (or trajectory) of $x$ under $f$ is the set $\left\{f^{n}(x)\right\}_{n \geq 0}$, where $f^{n}$ is the $n^{\text {th }}$ iterate of $f$. A sequence $\left\{y_{n}\right\}_{n \geq 0}$ is called a real orbit (or exact orbit) of $f$ if (RP) is satisfied for $n=0,1,2,3, \ldots$. We call such a sequence a "real" orbit to emphasize that there is no perturbation. For $\delta_{f}>0$ the sequence $\left\{x_{n}\right\}_{n \geq 0}$ is a $\delta_{f}$-pseudo orbit of $f$ if $\left|f\left(x_{n}\right)-x_{n+1}\right| \leqq \delta_{f}$ for every integer $n \geqq 0$. For $\delta_{x}>0$ the sequence $\left\{x_{n}\right\}_{n \geqq 0}$ will be $\delta_{x}$-shadowed by a sequence $\left\{y_{n}\right\}_{n \geqq 0}$ if $\left|x_{n}-y_{n}\right| \leqq \delta_{x}$ for all $n$.

When $f$ is sufficiently uniformly hyperbolic, Bowen [2] obtained a result saying that each pseudo orbit can be shadowed by a real orbit if the perturbation is small. More precisely, he gave conditions under which the following holds: for every $\delta_{x}>0$ there is an $\delta_{f}>0$ such that every $\delta_{f}$-pseudo orbit of $f$ in the nonwandering set can be $\delta_{x}$-shadowed by some real orbit of $f$.

We will study discrete processes which are not sufficiently hyperbolic to be modelled by an Axiom $A$ diffeomorphism. Let $f$ be a continuous map from the real line into itself. We assume that $f$ is strictly increasing on $(-\infty, c]$ and $f$ is strictly decreasing on $[c, \infty)$ for some real number $c$. Such a point $c$ will be called a critical point. A sequence $\left\{x_{n}\right\}_{n \geqq 0}$ is called non-critical if $x_{n} \neq c$ for each $n$, in other words, the sequence does not hit the critical point $c$. For each real $x$ we define $\operatorname{Symbol}(x)=L$ if $x<c, \operatorname{Symbol}(x)=C$ if $x=c$, and $\operatorname{Symbol}(x)=R$ if $x>c$. The itinerary (or symbolic sequence) of a sequence $\left\{x_{n}\right\}_{n \geq 0}$ is the sequence $\left\{\operatorname{Symbol}\left(x_{n}\right)\right\}_{n \geqq 0}$. Note that two non-critical sequences $\left\{x_{n}\right\}_{n \geqq 0}$ and $\left\{y_{n}\right\}_{n \geqq 0}$ have the same itinerary if they have the same symbolic sequences, i.e., $\left\{\operatorname{Symbol}\left(x_{n}\right)\right\}_{n \geq 0}=$ $\left\{\operatorname{Symbol}\left(y_{n}\right)\right\}_{n \geq 0}$. We frequently exclude consideration of orbits that pass through the critical point, because the kneading theory requires much more complicated terminology in such a situation. 
In this paper we will study two one-parameter families of maps, namely the one-parameter family $f_{\mu}$ of tent maps defined by

$$
f_{\mu}(x)= \begin{cases}\mu x & \text { if } \quad x \leqq c=\frac{1}{2} \\ \mu(1-x) & \text { if } \quad x \geqq c=\frac{1}{2}, \quad \mu>1 .\end{cases}
$$

as a prototype for the one-parameter family of expanding unimodal maps, and the one-parameter family $F_{\mu}$ of quadratic maps defined by

$$
F_{\mu}(x)=\mu x(1-x), \quad \mu>1,
$$

as a prototype for the one-parameter family of unimodal maps with negative Schwarzian derivative.

For either $f=f_{\mu}, 1<\mu<2$, or $f=F_{\mu}, 3 \leqq \mu<4$, we define the invariant set $\operatorname{Inv}\left(f, \delta_{f}\right)=\left[f\left(f(c)+\delta_{f}\right)-\delta_{f}, f(c)+\delta_{f}\right]$ for $\delta_{f} \geqq 0$ sufficiently small. This set has the property that if $x_{0}$ is in $\operatorname{Inv}\left(f, \delta_{f}\right)$ then so will be all $x_{n}, n \geqq 1$, whenever (NP) is satisfied with $\left|p_{n}\right| \leqq \delta_{f}$. No smaller interval will suffice for all choices of $\left\{p_{n}\right\}_{n \geqq 0}$.

We write $\delta_{x}$ for the distance between two nearby trajectories, $\delta_{\mu}$ for the increment of the parameter value, and $\delta_{f}$ for the maximum size of the perturbation. Write $g_{\mu}$ for either $f_{\mu}$ or $F_{\mu}$, if $\alpha=\mu+\delta_{\mu}$, then we write $g_{\alpha}=g_{\mu+\delta \mu}$.

Definition. For a given $\mu$ we say $g_{\mu}$ permits increased parameter shadowing if for each $\delta_{x}>0$ there is some $\delta_{\mu}>0$ and some $\delta_{f}>0$ so that each $\delta_{f}$-pseudo $g_{\mu}$-orbit starting in $\operatorname{Inv}\left(g_{\mu}, \delta_{f}\right)$ can be $\delta_{x}$-shadowed by a real $g_{\mu+\delta \mu}$-orbit, and $g_{\mu}$ permits same parameter shadowing if for each $\delta_{x}>0$ there is some $\delta_{f}>0$ such that every $\delta_{f}$-pseudo $g_{\mu}$-orbit starting in $\operatorname{Inv}\left(g_{\mu}, \delta_{f}\right)$ can be $\delta_{x}$-shadowed by a real $g_{\mu}$-orbit.

This study began by trying to show that increased parameter shadowing holds for the tent maps (Tent) with $1<\mu<2$. Two proofs emerged and the methods were quite general. One of these was based upon kneading sequences and turned out to be capable of also dealing with the quadratic map (Quad) with $1<\mu<4$. The other method, reported by Coven, Kan and Yorke [6], apparently cannot be used for (Quad) but can be used to show that same parameter shadowing holds for (Tent) for almost all $\mu$ in $(1,2)$. It is also shown that same parameter shadowing for (Tent) fails for an uncountable set of $\mu$ that is dense in $(1,2)$.

\section{Statement of the Results}

For clarity of exposition we will state the obtained results for noisy tent maps and noisy quadratic maps separately.

1-A. Noisy Tent Maps. Consider the 1-parameter family of maps on the real line given by $f_{\mu}(x)=\mu x$ for $x \leqq \frac{1}{2}, f_{\mu}(x)=\mu(1-x)$ for $x \geqq \frac{1}{2}$. We assume $1<\mu<$ $\mu+\delta_{\mu}<2$ and $0<\delta_{f}<\delta_{\mu}(\mu-1)^{2} /(4 \mu)$, and we define $\delta_{x}^{\text {tent }}=\left(\frac{1}{2} \delta_{\mu}+\delta_{f}\right) /\left(\mu+\delta_{\mu}-1\right)$. Notice $\delta_{x}^{\text {tent }}$ goes to zero as $\delta_{\mu}$ goes to zero.

Theorem A-1. For every non-critical $\delta_{f}$-pseudo $f_{\mu}$-orbit $\left\{x_{n}\right\}_{n \geq 0}$ with $x_{0}$ in $\operatorname{Inv}\left(f_{\mu}, \delta_{f}\right)$ there exists a real $f_{\mu+\delta \mu}$-orbit $\left\{y_{n}\right\}_{n \geqq 0}$ such that the itinerary of $\left\{y_{n}\right\}_{n \geqq 0}$ equals the itinerary of $\left\{x_{n}\right\}_{n \geqq 0}$. 
Theorem A-2. For every $\delta_{f}$-pseudo $f_{\mu}$-orbit $\left\{x_{n}\right\}_{n \geq 0}$ with $x_{0}$ in $\operatorname{Inv}\left(f_{\mu}, \delta_{f}\right)$ there exists a real $f_{\mu+\delta \mu}$-orbit $\left\{y_{n}\right\}_{n \geqq 0}$ such that $\left|x_{n}-y_{n}\right| \leqq \delta_{x}^{\text {tent }}$ for every nonnegative integer $n$.

Corollary. For each $\{\mu(n)\}_{n \geqq 0}, \mu-2 \delta_{f} \leqq \mu(n) \leqq \mu+2 \delta_{f}$ we have: For every $\left\{x_{n}\right\}_{n \geqq 0}$ given by $x_{n+1}=f_{\mu(n)}\left(x_{n}\right)$ with $x_{0}$ in $\operatorname{Inv}\left(f_{\mu}, \delta_{f}\right)$, there is $\left\{y_{n}\right\}_{n \geqq 0}$ with $y_{n+1}=f_{\mu+\delta \mu}\left(y_{n}\right)$, so that $\left|x_{n}-y_{n}\right| \leqq \delta_{x}^{\text {tent }}$ for each $n \geqq 0$.

Remark. $\mathrm{Th}^{m}$. A-2 says that $f_{\mu}$ permits increased parameter shadowing.

1-B. Noisy Quadratic Maps. Consider the 1-parameter family of maps on the real line defined by $F_{\mu}(x)=\mu x(1-x), \mu>1$. Our first result answers the question "Can the symbolic sequence of a given noisy orbit which does not hit the critical point also be realized by a real orbit?" For the quadratic map we assume $1<\mu<\mu+\delta_{\mu}<4$.

Theorem B-1. Assume that the critical point is not periodic and assume that $F_{\mu}$ has no one-sided attractive periodic orbit. Then there exists $\delta_{f}>0$ so that for each non-critical $\delta_{f}$-pseudo $F_{\mu}$-orbit $\left\{x_{n}\right\}_{n \geqq 0}$ with $x_{0}$ in $\operatorname{Inv}\left(F_{\mu}, \delta_{f}\right)$, there is a real $F_{\mu+\delta \mu}$-orbit $\left\{y_{n}\right\}_{n \geqq 0}$ such that the itinerary of $\left\{y_{n}\right\}_{n \geqq 0}$ equals the itinerary of $\left\{x_{n}\right\}_{n \geqq 0}$.

Remark. If $F_{\mu}$ has a non-critical attractive periodic orbit, then the conclusion in Theorem B-1 also holds for $\delta_{\mu}=0$.

For clarity, we give an example which shows that one cannot hope for a similar result as Theorem B-1 for all noisy sequences.

Example. Let $\mu$ be the parameter value so that $F_{\mu}^{3}\left(\frac{1}{2}\right)=\frac{1}{2}$. The itinerary of the real $F_{\mu}$-orbit $\left\{y_{n}\right\}_{n \geqq 0}$ with $y_{0}=\frac{1}{4} \mu$, is given by $R L C R L C R L C R L C R L C R L C \ldots$ Notice $F_{\mu}$ has an attractive periodic three orbit. Obviously, we can perturb the orbit slightly so that as many of the $C$ 's can be replaced by $R$ and $L$ as one desires. Hence, for each $\delta_{f}>0$ we can find a $\delta_{f}$-pseudo $F_{\mu}$-orbit whose itinerary is given by $R L L R L C R L L R L C R L C \ldots$ In contrast, for each $\delta_{\mu} \geqq 0$ there is no real $F_{\mu+\delta \mu}$-orbit whose itinerary starts with the second given sequence above. When the $C$ occurs for the second time, the itinerary of a real $F_{\mu+\delta \mu}$-orbit must thereafter repeat periodically, and this one does not. This completes the example.

An important question is "Is it true that every noisy orbit can be approximated by a real orbit for slightly greater parameter value?" First we will present an example for which the increased parameter shadowing does not hold. This example illustrates a shadowing obstacle that occurs whenever there is a one-sided attractive periodic orbit.

Example. Let $\mu$ be the saddle node bifurcation parameter value for the periodic three orbit. Choose $\delta_{\mu}>0$ such that the symbolic sequence of $F_{\mu+\delta \mu}\left(\frac{1}{2}\right)$ equals the symbolic sequence of $F_{\mu}\left(\frac{1}{2}\right)$, i.e., the kneading sequences of the maps $F_{\mu}$ and $F_{\mu+\delta \mu}$ are equal. This is true for all sufficiently small $\delta_{\mu}$.

For this value of $\mu$ there is a unique period three orbit. Let $q$ be the periodic point with period 3 which is nearest the critical point $\frac{1}{2}$. Under $F_{\mu}^{3}$ the point $q$ is 
a fixed point and it is attractive from the left, but it is repelling from the right. We define

$$
\delta_{x}^{*}=\frac{1}{2} \cdot \min \left\{\left|F_{\mu}^{k}(q)-F_{\mu}^{k}\left(\frac{1}{2}\right)\right|: 0 \leqq k \leqq 2\right\} .
$$

Let $\delta_{f}>0$ be given so that $\delta_{f}<(\mu-2)(4-\mu) /(4 \mu) \approx 0.02$ (this condition will become clear later on). Let $N \geqq 1$ be an integer such that $\left|F_{\mu}^{3 N}\left(\frac{1}{2}\right)-q\right|<\delta_{f}$. (Such an integer $N$ exists since, by a result due to Singer [32], the increasing sequence $\left\{F_{\mu}^{3 n}\left(\frac{1}{2}\right)\right\}_{n \geq 0}$, converges to $q$ as $n \rightarrow \infty$.) For $N$ sufficiently large, a perturbation of size $\delta_{f}>0$ will be large enough to push the trajectory past $q$.

We consider the noisy sequence $\left\{x_{n}\right\}_{n \geqq 0}: x_{0}=\frac{1}{2}, x_{n}=F_{\mu}\left(x_{n-1}\right)$ for $1 \leqq n \leqq 3 N-1$ and for $n \geqq 3 N+1$, and $x_{3 N}=F_{\mu}\left(x_{3 N-1}\right)+p_{3 N-1}$ with $0<p_{3 N-1}<\delta_{f}$ such that $x_{3 N}>q$ and $x_{3 N}$ is a periodic point of $F_{\mu}$. (The existence of such a real number $p_{3 N-1}$ is guaranteed by the fact that the periodic points are dense in the complement of the domain of attraction of the one-sided attractive periodic orbit, see Guckenheimer [13].)

Let $\left\{y_{n}\right\}_{n \geq 0}$ be a sequence defined by $y_{n+1}=F_{\mu+\delta \mu}\left(y_{n}\right)$ such that the symbolic sequence of $\left\{y_{n}\right\}_{n \geq 0}$ equals the symbolic sequence of $\left\{x_{n}\right\}_{n \geqq 1}$. From the kneading theory due to Milnor and Thurston [23] (see also Sect. 2-A), and a theorem due to Guckenheimer [13] it follows that such a sequence $\left\{y_{n}\right\}_{n \geqq 1}$ is uniquely determined.

For $\delta_{\mu}>0$ small, each trajectory of $F_{\mu+\delta \mu}$ that starts near $\frac{1}{2}$ will necessarily tend asymptotically to the attractive periodic 3 orbit. Hence, if $\left\{y_{n}\right\}_{n \geqq 1}$ has the same itinerary as our $\left\{x_{n}\right\}_{n \geq 1}$, it follows that $y_{0}$ must be far from $\frac{1}{2}$, so $\left|x_{0}-y_{0}\right|$ is not small and does not go to zero as $\delta_{\mu}$ goes to zero. More specifically, because of the fact that $\left\{y_{n}\right\}_{n \geqq 0}$ is contained in the complement of the domain of attraction of the attractive periodic orbit of $F_{\mu+\delta \mu}$, we have $\left|x_{0}-y_{0}\right|>\delta_{x}^{*}$. Even if $\delta_{f}$ goes to zero, the quantity $\delta_{x}^{*}$ is bounded away from zero. Consequently, the sequence $\left\{x_{n}\right\}_{n \geqq 0}$ cannot be $\delta_{x}^{*}$-shadowed. End of the example.

Now we will present the results obtained concerning the problem whether or not a noisy orbit can be approximated by a real orbit for slightly larger parameter value. It will turn out that increased parameter shadowing holds for all parameter values except for a countable set of parameter values. Consequently, the example given above is an exceptional case. Further we will see that, for a large open set $C$ of parameter values (those $\mu$ for which there is an attractive periodic orbit), one can actually shadow a given noisy $F_{\mu}$-orbit by a real $F_{\mu}$-orbit with $\mu$ in $C$. In particular, for such $\mu$ the parameter value need not be increased to find the real orbit for shadowing.

Theorem B-2. Assume that $F_{\mu}$ does not have a one-sided attractive periodic orbit. Then $F_{\mu}$ permits increased parameter shadowing. Moreover, if $F_{\mu}$ has an attractive periodic orbit, then $F_{\mu}$ permits same parameter shadowing.

Theorem B-3. Assume that $F_{\mu}$ has a one-sided attractive periodic orbit. Then there exists $\delta_{x}^{*}>0$ so that for every $\delta_{\mu} \geqq 0$ and every $\delta_{f}>0$ there is a $\delta_{f}$-pseudo $F_{\mu}$-orbit which cannot be $\delta_{x}^{*}$-shadowed by a real $F_{\mu+\delta \mu}$-orbit. 


\section{Preliminaries}

This section is devoted to symbolic dynamics. In the first subsection we will restrict our attention to reviewing those basic facts from kneading theory which are suitable for our purposes. In the second subsection we will, motivated by the concept of cylinder, introduce noisy cylinders. Let $f$ be a continuous map from an interval $[a, b], a<b$, into itself with one critical point $c$ in $(a, b)$ so that $f^{2}(c)<c<f(c)<b$. We assume that the endpoint $a$ is an unstable fixed point of $f$ and the point $b$ will be mapped into a, hence $f(a)=f(b)$; further we assume that $f$ is strictly increasing on $[a, c]$ with $f(x)>x$ for all $x, a<x \leqq c$ and $f$ is strictly decreasing on $[c, b]$.

2-A. Kneading Theory. Kneading theory for continuous piecewise monotone maps was largely developed by Milnor and Thurston [23], see also Derrida, Gervois and Pomeau [9,10], Guckenheimer [13, 14], Jonker [16], Collet and Eckmann [4], Guckenheimer and Holmes [15].

Previously, kneading theory has been used in order to obtain some desired results, e.g. (1) the classification of maps with one critical point by Guckenheimer [13], (2) the computation of the topological entropy of maps with one critical point by Collet, Crutchfield and Eckmann [5] and, (3) the bifurcation in one dimension by Jonker and Rand [17].

In this paper kneading theory will be used for the approximation of noisy orbits by real orbits. In order to do so, we shall review some basic facts. For each point $x, a \leqq x \leqq b$, the address $\operatorname{Symbol}(x)$ of $x$ is the formal symbol $L, C$, or $R$ as $x<c, x=c$, or $x>c$. The itinerary $I\left(f^{*}(x)\right)$ of $x$ under the map $f$ is the sequence of addresses $\left\{\operatorname{Symbol}\left(f^{n}(x)\right)\right\}_{n \geqq 0}$ of the successive images of $x$ under $f$. The sequence $\left\{\operatorname{Symbol}\left(f^{n}(x)\right)\right\}_{n \geq 0}$ will also be called the $f$-itinerary of $x$ as well as the itinerary of the sequence $\left\{f^{n}(x)\right\}_{n \geqq 0}$. We note that $I\left(f^{*}(f(x))\right)=s\left(I\left(f^{*}(x)\right)\right)$ with $s$ the shift map on sequences defined by $s\left(\left\{A_{n}\right\}_{n \geqq 0}\right)=\left\{A_{n+1}\right\}_{n \geqq 0}$. The kneading sequence $K(f)$ of $f$ is the $f$-itinerary of $f(c)$, i.e., $K(f)=\left\{\operatorname{Symbol}\left(f^{n}(f(c))\right)\right\}_{n \geq 0}=\{$ Symbol $\left.\left(f^{n}(c)\right)\right\}_{n \geqq 1}$. Finally, a finite sequence consisting of the symbols $L$ and $R$ is called odd or even as the number of $R$ 's it contains is odd or even.

Let $Y$ be either the set of $f$-itineraries (permitting the symbol $C$ ) or a subset of all sequences consisting only of the symbols $L$ and $R$. In either case we will define an ordering on $Y$. First, we define the order $L<C<R$ for the symbols $L, C$, and $R$. If $A=\left\{A_{n}\right\}_{n \geq 0}$ and $B=\left\{B_{n}\right\}_{n \geq 0}$ are two different elements of $Y$, then there is a smallest integer $N$ for which $A_{N} \neq B_{N}$. For $N=0$ we define $A<B$ if $A_{0}<B_{0}$; for $N \geqq 1$ we define $A<B$ if either (i) $\left\{A_{n}\right\}_{0 \leqq n \leqq N-1}\left(=\left\{B_{n}\right\}_{0 \leqq n \leqq N-1}\right)$ is even and $A_{N}<B_{N}$, or (ii) $\left\{A_{n}\right\}_{0 \leqq n \leqq N-1}\left(=\left\{B_{n}\right\}_{0 \leqq n \leqq N-1}\right)$ is odd and $B_{N}<A_{N}$. We will use the notation $A>B, A \leqq B$ and $A \geqq B$ in the standard way.

Now we will state the monotonicity theorem $M$ and the existence theorem $E$ due to Milnor and Thurston. For the proofs of these theorems we refer the reader to the mentioned references at the beginning of this section.

Theorem M. Let $x$ and $y$ be points in the interval $[a, b]$. Then (i) $x<y \Rightarrow I\left(f^{*}(x)\right) \leqq$ $I\left(f^{*}(y)\right)$; (ii) $I\left(f^{*}(x)\right)<I\left(f^{*}(y)\right) \Rightarrow x<y$.

Theorem E. Let $A=\left\{A_{n}\right\}_{n \geqq 0}$ be a sequence consisting of the symbols $L$ and $R$ such 
that $s(K(f)) \leqq\left\{A_{n+N}\right\}_{n \geq 0} \leqq K(f)$ for each integer $N \geqq 0$. Then there is a point $x$ in the interval $\left[f^{2}(c), f(c)\right]$ so that $A=I\left(f^{*}(x)\right)=\left\{\operatorname{Symbol}\left(f^{n}(x)\right)\right\}_{n \geqq 0}$.

2-B. Noisy Cylinders. We assume throughout this section that for given $\delta_{f} \geqq 0$ the set $\operatorname{Inv}\left(f, \delta_{f}\right)=\left[f\left(f(c)+\delta_{f}\right)-\delta_{f}, f(c)+\delta_{f}\right]$ has the property that if $x$ is in $\operatorname{Inv}\left(f, \delta_{f}\right)$ then $f(x)-\delta_{f}$ is in $\operatorname{Inv}\left(f, \delta_{f}\right)$ and $f(x)+\delta_{f}$ is in $\operatorname{Inv}\left(f, \delta_{f}\right)$. This will be true if $\delta_{f}$ is chosen sufficiently small.

Motivated by symbolic dynamics, see e.g. Parry [30], Lasota and Yorke [19], Alekseev and Jakobson [1] and, Crutchfield and Packard [8], we write for each $\delta_{f} \geqq 0$ :

$$
J\left\{L \mid\left(f, \delta_{f}\right)\right\}=[a, c), \quad J\left\{C \mid\left(f, \delta_{f}\right)\right\}=\{c\}, \quad J\left\{R \mid\left(f, \delta_{f}\right)\right\}=(c, b],
$$

and we define by induction for each nonnegative integer $n$, the noisy cylinder $J\left\{A_{n+1} A_{n} \ldots A_{0} \mid\left(f, \delta_{f}\right)\right\}$ by:

$$
\begin{aligned}
J\left\{A_{n+1} A_{n} \ldots A_{0} \mid\left(f, \delta_{f}\right)\right\}= & \left\{x \text { in } J\left\{A_{n+1} \mid\left(f, \delta_{f}\right)\right\}: \quad\left[f(x)-\delta_{f}, f(x)+\delta_{f}\right]\right. \\
& \left.\cap J\left\{A_{n} \ldots A_{0} \mid\left(f, \delta_{f}\right)\right\} \neq \emptyset\right\},
\end{aligned}
$$

with $A_{k}$ being one of the symbols $L, C$, or $R$ for each $k$ with $0 \leqq k \leqq n+1$.

Let $\left\{A_{n}\right\}_{0 \leqq n \leqq N}$ and $\left\{B_{n}\right\}_{0 \leqq n \leqq N}$ be two different finite sequences of the symbols $L, C$, and/or $R$. From the definition of noisy cylinders it follows that the noisy cylinder $J\left\{A_{N} \ldots A_{0} \mid\left(f, \delta_{f}\right)\right\}$ is either empty or a nonempty closed set. Hence, if $J\left\{A_{N} \ldots A_{0} \mid\left(f, \delta_{f}\right)\right\}$ is nonempty, then it is a point or a (nontrivial) interval. For $\delta_{f}=0$ we have that $J\left\{A_{n} \ldots A_{0} \mid\left(f, \delta_{f}\right)\right\} \cap J\left\{B_{N} \ldots B_{0} \mid\left(f, \delta_{f}\right)\right\}$ is empty, but for $\delta_{f}>0$ it can occur that $J\left\{A_{N} \ldots A_{0} \mid\left(f, \delta_{f}\right)\right\} \cap J\left\{B_{N} \ldots B_{0} \mid\left(f, \delta_{f}\right)\right\}$ is an interval.

Proposition 2-1. Let $\left\{A_{n}\right\}_{0 \leqq n \leqq N}$ be a given finite sequence consisting of the symbols $L, C$, and $R$. For $\delta_{f} \geqq 0$ we have:

(i) $J\left\{R A_{N} \ldots A_{0} \mid\left(f, \delta_{f}\right)\right\}=\emptyset \Rightarrow J\left\{L A_{N} \ldots A_{0} \mid\left(f, \delta_{f}\right)\right\}=\emptyset$,

(ii) $J\left\{L A_{N} \ldots A_{0} \mid\left(f, \delta_{f}\right)\right\} \neq \emptyset \Rightarrow J\left\{R A_{N} \ldots A_{0} \mid\left(f, \delta_{f}\right)\right\} \neq \emptyset$.

Proof. It follows from $f\left(f\left(f(c)+\delta_{f}\right)-\delta_{f}\right)-\delta_{f} \geqq f\left(f(c)+\delta_{f}\right)-\delta_{f}$.

Proposition 2-2. Let $\left\{A_{n}\right\}_{0 \leqq n \leqq N}$ be a given finite sequence consisting of the symbols $L, C$, and $R$. For $\delta_{f} \geqq 0$ we have: If $J\left\{A_{N} \ldots A_{0} \mid\left(f, \delta_{f}\right)\right\}$ is empty, then there is no $\delta_{f}$-pseudo $f$-orbit in $\operatorname{Inv}\left(f, \delta_{f}\right)$ whose itinerary has initial part $A_{N} \ldots A_{0}$.

Proof: Apply the definition of $J\left\{A_{N} \ldots A_{0} \mid\left(f, \delta_{f}\right)\right\}$.

Now we will state a monotonicity property for noisy cylinders. This property can be viewed as an analogue of the monotonicity theorem $M$ for the $f$-itineraries.

Proposition 2-3. Let $A=\left\{A_{n}\right\}_{n \geq 0}$ and $B=\left\{B_{n}\right\}_{n \geq 0}$ be two different sequences consisting of the symbols $L$ and $R$. Let $N$ be the smallest integer with $A_{N} \neq B_{N}$. Assume that the cylinders $J_{A}=J\left\{A_{0} \ldots A_{N} \mid\left(f, \delta_{f}\right)\right\}$ and $J_{B}=J\left\{B_{0} \ldots B_{N} \mid\left(f, \delta_{f}\right)\right\}$ are nonempty for some given $\delta_{f} \geqq 0$. Then we have:

(i) $\sup J_{A}<\sup J_{B} \Rightarrow A<B ; \quad$ (ii) $A<B \Rightarrow \sup J_{A} \leqq \sup J_{B}$;

(iii) $\inf J_{A}<\inf J_{B} \Rightarrow A<B ; \quad$ (iv) $A<B \Rightarrow \inf J_{A} \leqq \inf J_{B}$. 
Proof. The proof is straightforward, and it is left to the reader.

Proposition 2-4. Let $\left\{D_{n}\right\}_{0 \leqq n \leqq M}$ be an arbitrary given not empty sequence consisting of the symbols $L$ and $R$. Let $\left\{A_{n}\right\}_{0 \leqq n \leqq N}$ be a sequence consisting of L's and $R$ 's so that $\operatorname{Inf} J\left\{A_{0} \ldots A_{N} \mid\left(f, \delta_{f}\right)\right\}>f(c)+\delta_{f}$. Then we have: $J\left\{D_{0} \ldots D_{M} A_{0} \ldots A_{N} \mid\left(f, \delta_{f}\right)\right\}$ is empty.

Proof. From $\left\{x\right.$ in $\left.[a, b]:\left[f(x)-\delta_{f}, f(x)+\delta_{f}\right] \cap J\left\{A_{0} \ldots A_{N} \mid\left(f, \delta_{f}\right)\right\}\right\}=\emptyset$ it follows immediately that $J\left\{Y A_{0} \ldots A_{N} \mid\left(f, \delta_{f}\right)\right\}=\emptyset$ with $Y=L$ or $Y=R$.

Proposition 2-5. Let $\left\{A_{n}\right\}_{0 \leqq n \leqq N}$ be a finite sequence consisting of the symbols $L$ and $R$ such that inf $J\left\{A_{0} \ldots A_{N} \mid\left(f, \delta_{f}\right)\right\}>f(c)+\delta_{f}$. Then the cylinder $J\left\{R B_{0} \ldots B_{N} \mid\right.$ $\left.\left(f, \delta_{f}\right)\right\}$ is empty for each sequence $\left\{B_{n}\right\}_{0 \leqq n \leqq N}$ consisting of L's and $R$ 's with $\left\{B_{n}\right\}_{0 \leqq n \leqq N} \geqq\left\{A_{n}\right\}_{0 \leqq n \leqq N}$.

Proof. If $A_{n}=B_{n}$ for $0 \leqq n \leqq N$ then $J\left\{R B_{0} \ldots B_{N} \mid\left(f, \delta_{f}\right)\right\}$ is empty by Prop. 2-4. If $J\left\{B_{0} \ldots B_{N} \mid\left(f, \delta_{f}\right)\right\}$ is empty then $J\left\{R B_{0} \ldots B_{N} \mid\left(f, \delta_{f}\right)\right\}$ is empty. So we assume from now on $\left\{B_{n}\right\}_{0 \leqq n \leqq N}>\left\{A_{n}\right\}_{0 \leqq n \leqq N}$ and $J\left\{B_{0} \ldots B_{N} \mid\left(f, \delta_{f}\right)\right\}$ is nonempty. Then $\inf J\left\{B_{0} \ldots B_{N} \mid\left(f, \delta_{f}\right)\right\} \geqq \inf J\left\{A_{0} \ldots A_{N} \mid\left(f, \delta_{f}\right)\right\}$ by Prop. 2-3(iv). Using Prop. 2-4 we get $J\left\{R B_{0} \ldots B_{N} \mid\left(f, \delta_{f}\right)\right\}$ is empty.

\section{Tent Maps Under the Influence of Noise}

As in the introduction we let $f_{\mu}: R \rightarrow R$ be the one parameter family of maps defined by $f_{\mu}(x)=\mu x$ for $x \leqq \frac{1}{2}$ and $f_{\mu}(x)=\mu(1-x)$ for $x \geqq \frac{1}{2}$. Let $\mu$ be a fixed parameter value with $1<\mu<2$.

3-A. Tracks of Noisy Orbits. Let $\delta_{\mu}>0$ and $\delta_{f}>0$ be given. Let $\left\{x_{n}\right\}_{n \geq 0}$ be a $\delta_{f}$-pseudo $f_{\mu}$-orbit, i.e., $x_{n+1}=f_{\mu}\left(x_{n}\right)+p_{n}$ with $\left|p_{n}\right| \leqq \delta_{f}$. First, we will present a result concerning neighborhoods of the elements of the noisy sequence by applying $f_{\mu+\delta \mu}$.

Proposition 3-1. For every nonnegative integer $n$ we have: $\left[x_{n+1}-\delta_{x}, x_{n+1}+\delta_{x}\right] \cap$ $\left(-\infty, \frac{1}{2}\left(\mu+\delta_{\mu}\right)\right] \subset F_{\mu+\delta \mu}\left(\left[x_{n}-\delta_{x}, x_{n}+\delta_{x}\right]\right)$ with $\delta_{x}=\left(\frac{1}{2} \delta_{\mu}+\delta_{f}\right) /\left(\mu+\delta_{\mu}-1\right)$.

Proof. The proof is left to the reader.

Let $\delta_{x}$ be as in Proposition 3-1. We define for each integer $n \geqq 0$ the set $S_{0}(n)$ by $S_{0}(n)=\left[x_{n}-\delta_{x}, x_{n}+\delta_{x}\right]$. For every integer $n>0$ we define, by induction, for each integer $k$ with $0 \leqq k \leqq n-1, S_{k+1}(n)$ by

$$
S_{k+1}(n)=\left\{x \in S_{0}(n-k-1): \quad f_{\mu+\delta \mu}(x) \in S_{k}(n)\right\} .
$$

Proposition 3-2. If $S_{*}=\bigcap_{N \geqq 0} S_{N}(N) \neq \phi$, then there is a sequence $\left\{y_{n}\right\}_{n \geqq 0}$ defined by $y_{n+1}=f_{\mu+\delta \mu}\left(y_{n}\right)$ so that $\left|x_{n}-y_{n}\right| \leqq \delta_{x}$ for all $n$.

Proof. Assume that $S_{*}$ is a nonempty set. Pick arbitrarily $y_{0}$ in $S_{*}$. Then $\left|x_{0}-y_{0}\right| \leqq \delta_{x}$. Now define $\left\{y_{n}\right\}_{n \geq 0}$ by $y_{n+1}=f_{\mu+\delta \mu}\left(y_{n}\right)$.

Let integer $N>0$ be given. Fix arbitrarily an integer $n, 0 \leqq n \leqq N$. Then, by definition, we have that $y_{n}$ is in $S_{N-n}(N)$. From $S_{N-n}(N)$ included in $S_{0}(n)=$ $\left[x_{n}-\delta_{x}, x_{n}+\delta_{x}\right]$ it follows that $\left|x_{n}-y_{n}\right| \leqq \delta_{x}$. Since $n$ was arbitrary, we obtain 
that $\left|x_{n}-y_{n}\right| \leqq \delta_{x}$ for each integer $n, 0 \leqq n \leqq N$. The conclusion is that $\left|x_{n}-y_{n}\right| \leqq \delta_{x}$ for all $n$ since $N$ was arbitrary.

3-B. Noisy Attractor. A necessary condition, in order to be able to track noisy orbits which start in the unit interval, seems to be some invariance criterion, because the unit interval is positively $f_{\mu+\delta \mu}$-invariant for each $\delta_{\mu}$ with $0 \leqq \delta_{\mu} \leqq 2-\mu$. Of course, if such an invariance condition has been satisfied, this will not necessarily imply that every noisy orbit in the unit interval can be tracked by a real orbit.

The set $\operatorname{Inv}\left(f_{\mu}, 0\right)=\left[\mu-\frac{1}{2} \mu^{2}, \frac{1}{2} \mu\right]$ is a positively $f_{\mu}$-invariant set, and $f_{\mu}$ maps $\operatorname{Inv}\left(f_{\mu}, 0\right)$ onto itself. Consequently, $f_{\mu}\left(\operatorname{Inv}\left(f_{\mu}, 0\right)\right)=\operatorname{Inv}\left(f_{\mu}, 0\right)$. For each open set $U$ in $(0,1)$ containing $\operatorname{Inv}\left(f_{\mu}, 0\right)$ we have that $\bigcap_{k \geq 0} f_{\mu}^{k}(U)=\operatorname{Inv}\left(f_{\mu}, 0\right)$ since, for each $x, 0<x<1$, there is an integer $n \geqq 0$ such that $f_{\mu}^{n}(x)$ is in $\operatorname{Inv}\left(f_{\mu}, 0\right)$. Hence, $\operatorname{Inv}\left(f_{\mu}, 0\right)$ is an attractor for $f_{\mu}$.

The above observations indicate that for the noisy map $N\left\{f_{\mu}, \delta_{f}\right\}$ associated with $f_{\mu}$, also positively $N\left\{f_{\mu}, \delta_{f}\right\}$-invariant sets might exist with $N\left\{f_{\mu}, \delta_{f}\right\}(x)=$ $\left[f_{\mu}(x)-\delta_{f}, f_{\mu}(x)+\delta_{f}\right]$, for each integer $n \geqq 0$. Recall that $\operatorname{Inv}\left(f_{\mu}, \delta_{f}\right)=\left[f_{\mu}\left(f_{\mu}\left(\frac{1}{2}\right)+\right.\right.$ $\left.\left.\delta_{f}\right)-\delta_{f}, f_{\mu}\left(\frac{1}{2}\right)+\delta_{f}\right]$, and note that the attractor $\operatorname{Inv}\left(f_{\mu}, 0\right)$ of $f_{\mu}$ is contained in $\operatorname{Inv}\left(f_{\mu}, \delta_{f}\right)$ for every $\delta_{f} \geqq 0$. It will turn out that the set $\operatorname{Inv}\left(f_{\mu}, \delta_{f}\right)$ is positively $N\left\{f_{\mu}, \delta_{f}\right\}$-invariant, provided that $\delta_{f}$ is suitably chosen.

Proposition 3-3. For every $\delta_{f}$ with $0<\delta_{f} \leqq(\mu-1)(2-\mu) /(2 \mu)$ and for each $\delta_{f^{-}}$pseudo $f_{\mu}$-orbit $\left\{x_{n}\right\}_{n \geq 0}$ we have:

If $x_{0}$ is in $\operatorname{Inv}\left(f_{\mu}, \delta_{f}\right)$ then $x_{n}$ is in $\operatorname{Inv}\left(f_{\mu}, \delta_{f}\right)$ for all $n \geqq 0$.

Proof. By induction.

Proposition 3-4. For every $\delta_{f}$ with $0<\delta_{f}<(\mu-1)(2-\mu) /(2 \mu)$ the $\operatorname{set} \operatorname{Inv}\left(f_{\mu}, \delta_{f}\right)$ is an attractor for the noisy map $N\left\{f_{\mu}, \delta_{f}\right\}$.

Proof. Let $\delta_{f}$ be as in the proposition. For $x$ with $\delta_{f} /(\mu-1)<x<\frac{1}{2}$ we have $\mu x-\delta_{f}>x$ which implies inf $N\left\{f_{\mu}, \delta_{f}\right\}(x)>x$. Further, it is easily verified that $\delta_{f} /(\mu-1)<\mu-\frac{1}{2} \mu^{2}-\mu \delta_{f}-\delta_{f}$. We obtain that $\bigcap_{k \geq 0}\left(N\left\{f_{\mu}, \delta_{f}\right\}^{k}(U)=\operatorname{Inv}\left(f_{\mu}, \delta_{f}\right)\right.$ with $U=\left(\delta_{f} /(\mu-1), 1-\delta_{f} /(\mu-1)\right)$. We conclude: $\operatorname{Inv}\left(f_{\mu}, \delta_{f}\right)$ is an attractor for the noisy map $N\left\{f_{\mu}, \delta_{f}\right\}$.

3-C. Length of Noisy Cylinders. For our purposes we need the length of noisy cylinders. Let $\delta_{f} \geqq 0$ be given with $\delta_{f} \leqq(\mu-1)(2-\mu) /(2 \mu)$. For a given finite sequence $\left\{A_{n}\right\}_{0 \leqq n \leqq N}$ consisting of the symbols $L, C$, and $R$, the noisy cylinder $J\left\{A_{N} \ldots A_{0} \mid\left(f_{\mu}, \delta_{f}\right)\right\}$ is given by (2b). We write $\left|J\left\{A_{N} \ldots A_{0} \mid\left(f_{\mu}, \delta_{f}\right)\right\}\right|$ for the length of $J\left\{A_{N} \ldots A_{0} \mid\left(f_{\mu}, \delta_{f}\right)\right\}$.

Lemma 3-5. Let $\left\{A_{n}\right\}_{n \geq 0}$ be a sequence consisting of the symbols L, C, and R. For each $\delta_{J}>0$ there is a positive integer $N$ so that for each integer $n, n \geqq N$ we have: $\left|J\left\{A_{n} \ldots A_{0} \mid\left(f_{\mu}, \delta_{f}\right)\right\}\right| \leqq 2 \delta_{f} /(\mu-1)+\delta_{J}$.

Proof. Let $\left\{A_{n}\right\}_{n \geqq 0}$ be as in the proposition, and let $\delta_{J}>0$ be given. If $\delta_{J} \geqq 1$ then the claim is trivial. So, we assume that $\delta_{J}<1$. Choose $N$ so that $N \geqq-$ $\log \left(\delta_{J}\right) / \log (\mu)$. Then for each integer $n$ with $n \geqq N$ we have: 


$$
\begin{aligned}
\left|J\left\{A_{n} \ldots A_{0} \mid\left(f_{\mu}, \delta_{f}\right)\right\}\right| & \leqq\left|J\left\{A_{n-1} \ldots A_{0} \mid\left(f_{\mu}, \delta_{f}\right)\right\}\right| / \mu+2 \delta_{f} / \mu \\
& \leqq\left|J\left\{A_{0} \mid\left(f_{\mu}, \delta_{f}\right)\right\}\right| / \mu^{n}+\sum_{k=1}^{n} 2 \delta_{f} / \mu^{k} \leqq 1 /\left(2 \mu^{n}\right)+2 \delta_{f} \cdot \sum_{k=1}^{n} 1 / \mu^{k} \\
& \leqq \mu^{-n}+2 \delta_{f} /(\mu-1) \leqq \mu^{-N}+2 \delta_{f} /(\mu-1) \leqq \delta_{J}+2 \delta_{f} /(\mu-1) .
\end{aligned}
$$

Proposition 3-6. Let $\left\{A_{n}\right\}_{n \geqq 0}$ be a sequence consisting of the symbols $L, C$, and $R$. Assume that there exists a $\delta_{f}$-pseudo $f_{\mu}$-orbit $\left\{x_{n}\right\}_{n \geqq 0}$ with itinerary $\left\{A_{n}\right\}_{n \geqq 0}$, i.e., $\operatorname{Symbol}\left(x_{n}\right)=A_{n}$ for each $n$. Then the set of initial values for $\delta_{f}$-pseudo $f_{\mu}$-orbits with itinerary $\left\{A_{n}\right\}_{n \geqq 0}$ is a nonempty interval of length at most $2 \delta_{f} /(\mu-1)$.

\section{Proof. Apply Lemma 3-5.}

Remark. Let $\left\{A_{n}\right\}_{n \geqq 0}$ be the sequence defined by $A_{n}=R$ for each integer $n \geqq 0$. The interval consisting of initial values for $\delta_{f}$-pseudo $f_{\mu}$-orbits with itinerary $\left\{A_{n}\right\}_{n \geqq 0}$ has length $2 \delta_{f} /(\mu-1)$. Consequently, one cannot obtain a better estimate than the one given in Prop. 3-6.

3-D. Cylinders, Itineraries and Monotonicity. In this section we will study the question whether or not there is an ordering on the noisy cylinders in some sense, which is compatible with the ordering on the symbolic sequences. For the real number $\delta_{f} \geqq 0$ we assume that $\delta_{f} \leqq(\mu-1)(2-\mu) /(2 \mu)$. For a given finite sequence $\left\{A_{n}\right\}_{0 \leq n \leq N}$ consisting of the symbols $L, R$ the cylinder $J\left\{A_{N} \ldots A_{0} \mid\left(f_{\mu}, \delta_{f}\right)\right\}$ is given by (2b). For $\delta_{f}=0$ we have: if $x_{0}<y_{0}$ then $\left\{\operatorname{Symbol}\left(x_{n}\right)_{n \geqq 0}<\left\{\operatorname{Symbol}\left(y_{n}\right)\right\}_{n \geqq 0}\right.$ with $x_{n}=f_{\mu}^{n}\left(x_{0}\right), y_{n}=f_{\mu}^{n}\left(y_{0}\right)$, see e.g. Guckenheimer [13]. This monotonicity is not true for noisy sequences, but we have the following result.

Proposition 3-7. Let $\left\{x_{n}\right\}_{n \geqq 0}$ and $\left\{y_{n}\right\}_{n \geqq 0}$ be two non-critical $\delta_{f}$-pseudo $f_{\mu}$-orbits in $\operatorname{Inv}\left(f_{\mu}, \delta_{f}\right)$ with $x_{0}<y_{0}$. Then we have: if $y_{0}-x_{0}>2 \delta_{f} /(\mu-1)$ then S Symbol $\left.\left(x_{n}\right)\right\}_{n \geqq 0}<\left\{\operatorname{Symbol}\left(y_{n}\right)\right\}_{n \geqq 0}$.

Proof. Let $\left\{x_{n}\right\}_{n \geqq 0}$ and $\left\{y_{n}\right\}_{n \geqq 0}$ be as in the proposition. The itineraries of $\left\{x_{n}\right\}_{n \geqq 0}$ and $\left\{y_{n}\right\}_{n \geq 0}$ are sequences consisting of the symbols $L$ and $R$, because of the $\delta_{f}$-pseudo $f_{\mu}$-orbits are assumed to be non-critical. Applying Propositions 3-6 and 2-3 gives the result.

Proposition 3-8. Let $\left\{A_{n}\right\}_{n \geqq 0}$ be a sequence consisting of L's and R's. Assume that the cylinder $J\left\{A_{0} \ldots A_{N} \mid\left(f_{\mu}, \delta_{f}\right)\right\}$ is nonempty for some given integer $N>0$. Let $\left\{x_{n}\right\}_{n \geqq 0}$ be a non-critical $\delta_{f}$-pseudo $f_{\mu}$-orbit in $\operatorname{Inv}\left(f_{\mu}, \delta_{f}\right)$. Then inf $J\left\{A_{0} \ldots A_{N} \mid\left(f_{\mu}\right.\right.$, $\left.\left.\delta_{f}\right)\right\}>\frac{1}{2} \mu+\delta_{f}$ implies $\left\{\operatorname{Symbol}\left(x_{n}\right)\right\}_{n \geqq 0}<\left\{A_{n}\right\}_{n \geqq 0}$.

Proof. Let $\left\{A_{n}\right\}_{n \geqq 0}, \quad\left\{x_{n}\right\}_{n \geqq 0}$ and $N$ be as in the proposition. Assume $\inf J\left\{A_{0} \ldots A_{N} \mid\left(f_{\mu}, \delta_{f}\right)\right\}>\frac{1}{2} \mu+\delta_{f}$. We write $B_{n}=\operatorname{Symbol}\left(x_{n}\right), 0 \leqq n \leqq N$. Since $\left\{x_{n}\right\}_{n \geq 0}$ in $\operatorname{Inv}\left(f_{\mu}, \delta_{f}\right)$ we know inf $J\left\{B_{0} \ldots B_{N} \mid\left(f_{\mu}, \delta_{f}\right)\right\}<\frac{1}{2} \mu+\delta_{f}$. Applying Prop. 2-3 (iii) gives $\left\{B_{n}\right\}_{n \geqq 0}<\left\{A_{n}\right\}_{n \geqq 0}$. We conclude: $\left\{\operatorname{Symbol}\left(x_{n}\right)\right\}_{n \geqq 0}<\left\{A_{n}\right\}_{n \geqq 0}$.

3-E. Pseudo Orbits and Kneading Sequence. Choose $\delta_{\mu}$ so that $1<\mu<\mu+\delta_{\mu}<2$. Choose the noise level $\delta_{f}$ so that $0<\delta_{f}<\delta_{\mu}(\mu-1)^{2} /(4 \mu)$. For a given finite sequence $\left\{A_{n}\right\}_{0 \leqq n \leqq N}$ consisting of the symbols $L, C$, and $R$ the cylinder $J\left\{A_{N} \ldots A_{0} \mid\left(f_{\mu}, \delta_{f}\right)\right\}$ is defined by (2b). The kneading sequence $K\left(f_{\mu}\right)$ of $f_{\mu}$ is the $f_{\mu}$-itinerary of $f_{\mu}\left(\frac{1}{2}\right)=\frac{1}{2} \mu$. Let $\left\{x_{n}\right\}_{n \geqq 0}$ be a non-critical $\delta_{f}$-pseudo $f_{\mu}$-orbit with $x_{0}$ in $\operatorname{Inv}\left(f_{\mu}, \delta_{f}\right)$. 
In this section we will obtain the result that $\left\{\operatorname{Symbol}\left(x_{n}\right)\right\}_{n \geqq 0}<K\left(f_{\mu+\delta \mu}\right)$ with $\left\{\operatorname{Symbol}\left(x_{n}\right)\right\}_{n \geqq 0}$ the itinerary of the sequence $\left\{x_{n}\right\}_{n \geqq 0}$. In order to prove this claim, we will introduce an auxiliary map $g$ being topologically conjugate with $f_{\mu+\delta \mu}$, for which we will show that $\left\{\operatorname{Symbol}\left(x_{n}\right)\right\}_{n \geqq 0}<K(g)=K\left(f_{\mu+\delta \mu}\right)$. Then by the existence Theorem $\mathrm{E}$ due to Milnor and Thurston we know that there exists a sequence $\left\{z_{n}\right\}_{n \geqq 0}$ defined by $z_{n+1}=g\left(z_{n}\right)$ such that the itinerary of the sequence $\left\{z_{n}\right\}_{n \geqq 0}$ equals the itinerary of the sequence $\left\{x_{n}\right\}_{n \geqq 0}$, i.e., $\operatorname{Symbol}\left(z_{n}\right)=\operatorname{Symbol}\left(x_{n}\right)$ for each $n$.

Let the diffeomorphism $\Phi$ from $[0,1]$ into $[s, 1-s]$ be defined by $\Phi(x)=$ $(1-2 s) x+s$, with $s=\left(\frac{1}{2} \delta_{\mu}-\delta_{f}\right) /\left(\mu+\delta_{\mu}-1\right)$. We consider the map $g:[s, 1-s] \rightarrow$ $[s, 1-s]$ defined by $g(x)=\Phi \circ f_{\mu+\delta \mu} \circ \Phi^{-1}(x)$.

Proposition 3-9. The map $g$, which is topologically conjugate with $f_{\mu+\delta \mu}$, has the following properties:

(i) $g\left(\frac{1}{2}\right)=\frac{1}{2} \mu+\delta_{f}$; (ii) $g\left(\mu-\frac{1}{2} \mu^{2}-\mu \delta_{f}-\delta_{f}\right)<\mu^{2}-\frac{1}{2} \mu^{3}-\mu^{2} \delta_{f}-\mu \delta_{f}-\delta_{f}$; and (iii) $g\left(\frac{1}{2} \mu+\delta_{f}\right)+2 \delta_{f} /(\mu-1)<\mu-\frac{1}{2} \mu^{2}-\mu \delta_{f}-\delta_{f}$.

Proof. Notice $g(x)=f_{\mu+\delta \mu}(x)-\left(\frac{1}{2} \delta_{\mu}-\delta_{f}\right)$ for $s \leqq x \leqq 1-s$. The rest of the proof is straightforward, and it is left to the reader.

Proposition 3-10. $\left\{\operatorname{Symbol}\left(x_{n}\right)\right\}_{n \geqq 0}<K(g)$.

Proof. By Prop. 3-5 we know that there exists an integer $M>0$ such that the length of the cylinder $J\left\{A_{M} \ldots A_{0} \mid\left(f_{\mu}, \delta_{f}\right)\right\}$ is smaller than $2 \delta_{f} /(\mu-1)+\frac{1}{2}\{\mu-$ $\left.\frac{1}{2} \mu^{2}-\mu \delta_{f}-\delta_{f}-2 \delta_{f} /(\mu-1)-g\left(\frac{1}{2} \mu+\delta_{f}\right)\right\}$ with $\left\{A_{n}\right\}_{0 \leqq n \leqq M}$ an arbitrary finite sequence consisting of the symbols $L$ and $R$. Pick $\alpha$ in $\left(g\left(\frac{1}{2} \mu+\delta_{f}\right), g\left(\frac{1}{2} \mu+\delta_{f}\right)+\right.$ $\left.\frac{1}{2}\left\{\mu-\frac{1}{2} \mu^{2}-\mu \delta_{f}-\delta_{f}-2 \delta_{f} /(\mu-1)-g\left(\frac{1}{2} \mu+\delta_{f}\right)\right\}\right)$ such that $f_{\mu}^{N}(\alpha)=\frac{1}{2}$ for some integer $N \geqq M$. The existence of such a point $\alpha$ is guaranteed by the well known fact that the union of the pre-images of the critical point (i.e. the set $\left\{x: f_{\mu}^{n}(x)=\frac{1}{2}\right.$, $n \geqq 0\})$ is dense in the interval $[0,1]$. Note that this fact also follows from Prop. 3-5 with $\delta_{f}=0$.

We write $D_{n}=\operatorname{Symbol}\left(f_{\mu}^{n}(\alpha)\right)$ for each integer $n \geqq 0$. Notice $D_{N}=C$. Since $K\left(f_{\mu}\right)<K\left(f_{\mu+\delta \mu}\right)$ we know by the existence Theorem $\mathrm{E}$ of Milnor and Thurston that there is a point $\beta$ in the interval $\left(g\left(\frac{1}{2} \mu+\delta_{f}\right), \frac{1}{2}\right)$ such that Symbol $\left(f_{\mu+\delta \mu}{ }^{n}(\beta)=D_{n}\right.$ for each $n, 0 \leqq n \leqq N$.

From the choice of $\alpha$ and the definition of cylinders we get inf $J\left\{R D_{0} \ldots D_{N} \mid\right.$ $\left.\left(f_{\mu}, \delta_{f}\right)\right\}>\frac{1}{2} \mu+\delta_{f}$. By applying Prop. 3-8 we conclude that $\left\{\operatorname{Symbol}\left(x_{n}\right)\right\}_{n \geqq 0}<$ $K(g)$.

Proposition 3-11. There exists a sequence $\left\{z_{n}\right\}_{n \geqq 0}$ defined by $z_{n+1}=g\left(z_{n}\right)$ so that $\operatorname{Symbol}\left(z_{n}\right)=\operatorname{Symbol}\left(x_{n}\right)$ for each integer $n \geqq 0$.

Proof. Apply Prop. 3-10 and the Theorem E of Milnor and Thurston.

3-F. Proof of the Results. Let $f_{\mu}: R \rightarrow R$ be defined by $f_{\mu}(x)=\mu x$ for $x \leqq \frac{1}{2}$ and $f_{\mu}(x)=\mu(1-x)$ for $x \geqq \frac{1}{2}$. We assume that $1<\mu<\mu+\delta_{\mu}<2$ and $0<\delta_{f}<\delta_{\mu}$. $(\mu-1)^{2} /(4 \mu)$; and we recall that $\delta_{x}=\left(\frac{1}{2} \delta_{\mu}+\delta_{f}\right) /\left(\mu+\delta_{\mu}-1\right)$, and $\operatorname{Inv}\left(f_{\mu}, \delta_{f}\right)=$ $\left[f_{\mu}\left(f_{\mu}\left(\frac{1}{2}\right)+\delta_{f}\right)-\delta_{f}, f_{\mu}\left(\frac{1}{2}\right)+\delta_{f}\right]$.

Proof of Theorem $A-1$. Let $\left\{x_{n}\right\}_{n \geqq 0}$ be a non-critical $\delta_{f}$-pseudo $f_{u}$-orbit with $x_{0}$ 
in $\operatorname{Inv}\left(f_{\mu}, \delta_{f}\right)$. By Prop. 3-3 we know that $\left\{x_{n}\right\}_{n \geqq 0}$ is in $\operatorname{Inv}\left(f_{\mu}, \delta_{f}\right)$. Let the maps $g$ and $\Phi$ be as in Sect. 3-E. Applying Prop. 3-11 we obtain that there exists a real $g$-orbit $\left\{z_{n}\right\}_{n \geqq 0}$ with $\left\{\operatorname{Symbol}\left(z_{n}\right)\right\}_{n \geqq 0}=\left\{\operatorname{Symbol}\left(x_{n}\right)\right\}_{n \geqq 0}$. We define $y_{n}=\Phi^{-1}\left(z_{n}\right)$, for each $n \geqq 0$. We conclude: the sequence $\left\{y_{n}\right\}_{n \geq 0}$ is a real $f_{\mu+\delta \mu}$-orbit with $\left\{\operatorname{Symbol}\left(y_{n}\right)\right\}_{n \geqq 0}=\left\{\operatorname{Symbol}\left(x_{n}\right)\right\}_{n \geqq 0}$.

Proof of Theorem A-2. Let $\left\{x_{n}\right\}_{n \geqslant 0}$ be a non-critical $\delta_{f}$-pseudo $f_{\mu}$-orbit with $x_{0}$ in $\operatorname{Inv}\left(f_{\mu}, \delta_{f}\right)$. Then, by Prop. 3-3, $\left\{x_{n}\right\}_{n \geqq 0}$ is in $\operatorname{Inv}\left(f_{\mu}, \delta_{f}\right)$. Let for each integer $N \geqq 0$ the set $S_{k}(N), 0 \leqq k \leqq N$, be defined as in Sect. 3-A. For every integer $n \geqq 0$ we have by Prop. 3-1 $\left[x_{n+1}-\delta_{x}, x_{n+1}+\delta_{x}\right] \cap\left(-\infty,\left(\mu+\delta_{\mu}\right) / 2\right] \subset f_{\mu+\delta_{\mu}}\left(\left[x_{n}-\right.\right.$ $\left.\left.\delta_{x}, x_{n}+\delta_{x}\right]\right)$. Let $\left\{y_{n}\right\}_{n \geq 0}$ be the real $f_{\mu+\delta \mu}$-orbit whose itinerary equals that of $\left\{x_{n}\right\}_{n \geqq 0}$, such a real orbit exists by Theorem A-1. It follows that $S_{*}=\bigcap_{N \geqq 0} S_{N}(N)$ is nonempty since $y_{0}$ is in $S_{*}$. Using Prop. 3-2 gives $\left|x_{n}-y_{n}\right| \leqq \delta_{x}$ for each $n \geqq 0$.

Proof of Corollary. The union of the graphs of $f_{\mu(n)}$ is contained in the graph of the noisy map, more precisely: the set $\left\{\left(x, f_{\mu(n)}(x)\right): x\right.$ in $\left.\operatorname{Inv}\left(f_{\mu}, \delta_{f}\right)\right\}$ is in $\left\{\left(x,\left[f_{\mu}(x)-\delta_{f}, f_{\mu}(x)+\delta_{f}\right]\right): x\right.$ in $\left.\operatorname{Inv}\left(f_{\mu}, \delta_{f}\right)\right\}$.

\section{Quadratic Maps with Noise}

Let $F_{\mu}: R \rightarrow R$ be the one-parameter family of maps defined by $F_{\mu}(x)=\mu x(1-x)$. We restrict $\mu$ to $3 \leqq \mu<4$, except where specified.

4-A. Noisy Attractor. The set $\operatorname{Inv}\left(F_{\mu}, 0\right)=\left[F_{\mu}^{2}\left(\frac{1}{2}\right), F_{\mu}\left(\frac{1}{2}\right)\right]$ is an attractor for $F_{\mu}$, because $F_{\mu}\left(\operatorname{Inv}\left(F_{\mu}, 0\right)\right)=\operatorname{Inv}\left(F_{\mu}, 0\right)$ and for each open $U$ in $(0,1)$ containing $\operatorname{Inv}\left(F_{\mu}, 0\right)$ we have $\bigcap_{k \geq 0} F_{\mu}^{k}(U)=\operatorname{Inv}\left(F_{\mu}, 0\right)$.

For every $\delta_{f} \geqq \overline{0}$ the noisy map $N\left\{F_{\mu}, \delta_{f}\right\}$ is defined by $N\left\{F_{\mu}, \delta_{f}\right\}(x)=$ $\left[F_{\mu}(x)-\delta_{f}, F_{\mu}(x)+\delta_{f}\right]$. As for the tent map we will see that an attractor will exist provided that the noise level $\delta_{f}$ is sufficiently small. Recall $\operatorname{Inv}\left(F_{\mu}, \delta_{f}\right)=\left[F_{\mu}\left(F_{\mu}\left(\frac{1}{2}\right)+\right.\right.$ $\left.\left.\delta_{f}\right)-\delta_{f}, F_{\mu}\left(\frac{1}{2}\right)+\delta_{f}\right]$. We will assume for the rest of this section that $0<\delta_{f}<(\mu-2)$ $(4-\mu) /(4 \mu)$.

Proposition 4-1. For each $\delta_{f}$-pseudo $F_{\mu}$-orbit $\left\{x_{n}\right\}_{n \geq 0}$ we have: if $x_{0}$ is in $\operatorname{Inv}\left(F_{\mu}, \delta_{f}\right)$ then the sequence $\left\{x_{n}\right\}_{n \geq 0}$ is in $\operatorname{Inv}\left(F_{\mu}, \delta_{f}\right)$.

Proof. By induction.

Proposition 4-2. $\operatorname{Inv}\left(F_{\mu}, \delta_{f}\right)$ is an attractor for $N\left\{F_{\mu}, \delta_{f}\right\}$.

Proof. Write $\operatorname{rad}=\left[(\mu-1)^{2}-4 \mu \delta_{f}\right]^{1 / 2}$. For $x=(\mu-1-\operatorname{rad}) /(2 \mu)$ we have $\mu x(1-x)-\delta_{f}=x$, and for each point $x$ with $(\mu-1-\operatorname{rad}) /(2 \mu)<x<\frac{1}{2}$ we have $\mu x(1-x)-\delta_{f}>x$; this implies $\inf N\left\{F_{\mu}, \delta_{f}\right\}(x)>x$. Since $\mu \alpha(1-\alpha)>\alpha$ with $\alpha=\mu\left(\frac{1}{4} \mu+\delta_{f}\right)\left(1-\frac{1}{4} \mu-\delta_{f}\right)-\delta_{f} \quad$ we have $\quad(\mu-1-\operatorname{rad}) /(2 \mu)<\mu\left(\frac{1}{4} \mu+\delta_{f}\right)$ $\left(1-\frac{1}{4} \mu-\delta_{f}\right)$. We obtain, by writing $U=(\beta, 1-\beta)$ with $\beta=(\mu-1-\operatorname{rad}) /(2 \mu)$, that $\bigcap_{k \geq 0}\left(N\left\{F_{\mu}, \delta_{f}\right\}\right)^{k}(U)=\operatorname{Inv}\left(F_{\mu}, \delta_{f}\right)$. The conclusion is that $\operatorname{Inv}\left(F_{\mu}, \delta_{f}\right)$ is an attractor for $N\left\{F_{\mu}, \delta_{f}\right\}$.

4-B. Noisy Periodic Attractors and Shadowing. We assume that $F_{\mu}$ has an attractive 
periodic orbit $P$. Hence, there exists a smallest positive integer $p$ such that $P$ consists of $p$ asymptotically stable periodic points $q_{i}$ with $F_{\mu}\left(q_{i}\right)=q_{i+1}, 1 \leqq i \leqq p-1$ and $F_{\mu}\left(q_{p}\right)=q_{1}$. Since $F_{\mu}^{\prime}(0)=\mu>1$, it follows from a theorem due to Singer [31] that all the other periodic points are not asymptotically stable.

First, we have to define "Noisy periodic attractor." A closed set $A$ is called an attractor for the map $N\left\{F_{\mu}, \delta_{f}\right\}$ (which is defined by $N\left\{F_{\mu}, \delta_{f}\right\}(x)=\left[F_{\mu}(x)-\right.$ $\left.\left.\delta_{f}, F_{\mu}(x)+\delta_{f}\right]\right)$ if there exists an open neighborhood $U$ of $A$ such that $\bigcap_{k \geq 0}\left(N\left\{F_{\mu}, \delta_{f}\right\}\right)^{k}(U)=A$. An attractor $A$ for the noisy map $N\left\{F_{\mu}, \delta_{f}\right\}$ is called a noisy periodic attractor if the following two conditions are satisfied: (i) The attractor $A$ contains the attractive periodic orbit $P$ of $F_{\mu}$; (ii) Each component of $A$ contains precisely one point of $P$. Such a noisy periodic attractor for the noisy map $N\left\{F_{\mu}, \delta_{f}\right\}$ will be denoted by $A_{p}\left(F_{\mu}, \delta_{f}\right)$.

Proposition 4-3. There exists $\delta_{f}>0$ such that $N\left\{F_{\mu}, \delta_{f}\right\}$ has a noisy periodic attractor.

Proof. It follows immediately from the proof of Theorem B in [29].

Proposition 4-4. If the attractive periodic orbit $P$ of $F_{\mu}$ does not contain the critical point of $F_{\mu}$, then there exists $\delta_{f}>0$ so that $N:\left\{F_{\mu}, \delta_{f}\right\}$ has a noisy periodic attractor $A_{p}\left(f_{\mu}, \delta_{f}\right)$ for which we have: The itinerary of each $\delta_{f}$-pseudo $F_{\mu}$-orbit starting in the component of $A_{p}\left(F_{\mu}, \delta_{f}\right)$ which contains the supremum of the noisy periodic attractor, equals the kneading sequence $K\left(F_{\mu}\right)$ of $F_{\mu}$.

Proof. It follows immediately from the proof of Theorem D in [29].

Proposition 4-5. If the attractive periodic orbit $P$ of $F_{\mu}$ does not contain the critical point of $F_{\mu}$, then there exists $\delta_{f}>0$ so that for every non-critical $\delta_{f}$-pseudo $F_{\mu}$-orbit $\left\{x_{n}\right\}_{n \geqq 0}$ in $\operatorname{Inv}\left(F_{\mu}, \delta_{f}\right)$, we have: $\left\{\operatorname{Symbol}\left(x_{n}\right)\right\}_{n \geqq 0} \leqq K\left(F_{\mu}\right)$, with $K\left(F_{\mu}\right)$ the kneading sequence of $F_{\mu}$.

Proof. It follows from the Propositions 4-3, 4-4, and 2-3 (i) above; and the proofs of Theorems B and D in [29].

Notice in Prop. 4-5 we have the restriction that $\delta_{f}$ goes to zero as the parameter goes to some critical value for which the critical point is periodic. However, the same parameter shadowing will be permitted in such a situation for some $\delta_{f}$ bounded away from zero.

Therefore, we will say, a $\delta_{f}$-pseudo $F_{\mu}$-orbit $\left\{x_{n}\right\}_{n \geq 0}$ is bounded away from the noisy attractor $A_{p}\left(F_{\mu}, \delta_{f}\right)$ if the distance between $A_{p}\left(F_{\mu}, \delta_{f}\right)$ and the closure of $\left\{x_{n}\right\}_{n \geqq 0}$ is bounded away from zero. Obviously, for $\delta_{f}$ as in Proposition 4-3 we have: if a $\delta_{f}$-pseudo $F_{\mu}$-orbit $\left\{x_{n}\right\}_{n \geqq 0}$ is not bounded away from the noisy attractor, then it will be attracted by the noisy attractor.

Proposition 4-6. For every $\delta_{x}>0$ there exists $\delta_{f}>0$ such that each $\delta_{f}$-pseudo $F_{\mu}$-orbit in $\operatorname{Inv}\left(F_{\mu}, \delta_{f}\right)$ can be $\delta_{x}$-shadowed by some real $F_{\mu}$-orbit.

Proof. It follows from Proposition 4-5 above, the existence Theorem E due to Milnor and Thurston, and the proofs of Theorems B and D in [29].

4-C. Obstruction for Shadowing. In this section we assume that $\mu$ is a saddle-node 
(or tangent) bifurcation parameter value. Assume that $F_{\mu}$ has a one-sided attractive periodic orbit $P$ consisting of $p$ periodic points with smallest period $p$ for some integer $p$. Notice $p \geqq 3$.

Proposition 4-7. There exists $\delta_{x}^{*}>0$ such that for every $\delta_{\mu} \geqq 0$ and each $\delta_{f}>0$ there is a non-critical $\delta_{f}$-pseudo $F_{\mu}$-orbit $\left\{x_{n}\right\}_{n \geqq 0}$ which cannot be $\delta_{x}^{*}$-shadowed by a real $F_{\mu+\delta \mu}$-orbit, whose itinerary equals that of $\left\{x_{n}\right\}_{n \geqq 0}$.

Proof. It is no restriction to assume that $\mu \leqq \mu+\delta_{\mu} \leqq 4$ and that $0<\delta_{f}<$ $(\mu-2)(4-\mu) /(4 \mu)$. Let $q$ be a point of $P$ such that $\left|q-\frac{1}{2}\right|=\min \left\{\left|x-\frac{1}{2}\right|: x\right.$ in $\left.P\right\}$. For $\delta_{\mu}>0$ sufficiently small we have that $F_{\mu+\delta \mu}$ has an attractive periodic orbit $P_{s}$ and a repelling periodic orbit $P_{u}$ both emerging from $P$ at parameter value $\mu$. We write $q\left(\mu+\delta_{\mu}\right)$ in $P_{u}$ such that $\left|q\left(\mu+\delta_{\mu}\right)-\frac{1}{2}\right|=\min \left\{\left|x-\frac{1}{2}\right|: x\right.$ in $\left.P_{u}\right\}$.

For each $\delta_{\mu} \geqq 0$ the periodic point $q\left(\mu+\delta_{\mu}\right)$ exists by a result due to Milnor and Thurston which says that the kneading sequence is non-decreasing for the quadratic family, see Milnor [24]. We define $\delta_{x}^{*}=\frac{1}{2} \cdot \inf \left\{\left|q\left(\mu+\delta_{\mu}\right)-\frac{1}{2}\right|: \mu \leqq \mu+\right.$ $\left.\delta_{\mu} \leqq 4\right\}$, since $q$ depends continuously on the parameter. Let $N>0$ be an integer so that $\left|F_{\mu}^{p N}\left(\frac{1}{2}\right)-q\right|<\delta_{f}$. Such an integer $N$ exists by a result due to Singer [32] since $F_{\mu}^{\prime}(0)>1$, which says that $\lim _{n \rightarrow \infty} F_{\mu}^{p n}\left(\frac{1}{2}\right)=q$.

Consider the $\delta_{f}$-pseudo $F_{\mu}$-orbit $\left\{x_{n}\right\}_{n \geqq 0}$ defined as follows: $x_{0}=\frac{1}{2}, x_{n}=$ $F_{\mu}\left(x_{n-1}\right)$ for $1 \leqq n \leqq p N-1$ and $n \geqq p N+1$, and $x_{p N}=F_{\mu}\left(x_{p N-1}\right)-\alpha$ if $F_{\mu}^{p}$ has a local maximum at $\frac{1}{2}$ and $x_{p N}=F_{\mu}\left(x_{p N-1}\right)+\alpha$ if $F_{\mu}^{p}$ has a local minimum at $\frac{1}{2}$ with $0<\alpha \leqq \delta_{f}$ such that $\left|x_{p N}-\frac{1}{2}\right|>\left|q-\frac{1}{2}\right|$ and $x_{p N}$ periodic. The existence of such a real number $\alpha$ is guaranteed by the fact that the periodic points are dense in the complement of the domain of attraction of $P$, see e.g. Guckenheimer [13].

Let $\delta_{\mu} \geqq 0$ be given and let $\left\{y_{n}\right\}_{n \geq 0}$ be a sequence in $[0,1]$ defined by $y_{n+1}=F_{\mu+\delta \mu}\left(y_{n}\right)$ such that the itinerary of $\left\{y_{n}\right\}_{n \geq 1}$ equals the itinerary of $\left\{x_{n}\right\}_{n \geqq 1}$. From the kneading theory due to Milnor and Thurston [23] and from a theorem due to Guckenheimer [13] it follows that $\left\{y_{n}\right\}_{n \geqq 1}$ is uniquely determined.

Because of the fact that $\left|y_{0}-\frac{1}{2}\right|>\left|q\left(\mu+\delta_{\mu}\right)-\frac{1}{2}\right|>\delta_{x}^{*}$ we have $\left|x_{0}-y_{0}\right|>\delta_{x}^{*}$. Now we consider the noisy sequence $\left\{X_{n}\right\}_{n \geqq 0}$ defined by $X_{0}=x_{0}+\delta_{f} / 100$ if $y_{0}>\frac{1}{2}, X_{0}=x_{0}-\delta_{f} / 100$ if $y_{0}<\frac{1}{2}$, and $X_{n}=x_{n}$ for $n \geqq 1$.

Conclusion. The $\delta_{f}$-pseudo $F_{\mu}$-orbit $\left\{X_{n}\right\}_{n \geqq 0}$ cannot be $\delta_{x}^{*}$-shadowed by a real $F_{\mu+\delta \mu}$-orbit for every $\delta_{\mu} \geqq 0, \mu+\delta_{\mu} \leqq 4$.

4-D. When There are only Repelling Periodic Points. We assume that all the periodic points of $F_{\mu}$ are repelling. For each sequence $\left\{A_{n}\right\}_{n \geq 0}$ consisting of the symbols $L, C$, and/or $R$ the noisy cylinder $J\left\{A_{0} \ldots A_{N} \mid\left(F_{\mu}, \delta_{f}\right)\right\}$ is defined in (2b) for each $N \geqq 0$. The kneading sequence $K\left(F_{\mu}\right)$ of $F_{\mu}$ is the itinerary of $F_{\mu}\left(\frac{1}{2}\right)$.

In this section we will obtain the result that for $\delta_{\mu}>0$ there is $\delta_{f}>0$ such that for every non-critical $\delta_{f}$-pseudo $F_{\mu}$-orbit $\left\{x_{n}\right\}_{n \geqq 0}$ we have $\left\{\operatorname{Symbol}\left(x_{n}\right)\right\}_{n \geqq 0} \leqq$ $K\left(F_{\mu+\delta \mu}\right)$. In order to prove such a claim, we will similarly as with the tent maps, introduce an auxiliary map $g$ being topologically conjugate with $F_{\mu+\delta \mu}$ for which we will show that $\left\{\operatorname{Symbol}\left(x_{n}\right)\right\}_{n \geqq 0} \leqq K(g)=K\left(F_{\mu+\delta \mu}\right)$.

Proposition 4-8. For every $\delta_{\mu}>0$ there exists $\delta_{f}>0$ such that for each non-critical $\delta_{f}$-pseudo $F_{\mu}$-orbit $\left\{x_{n}\right\}_{n \geqq 0}$ in $\operatorname{Inv}\left(F_{\mu}, \delta_{f}\right)$ we have: $\left\{\operatorname{Symbol}\left(x_{n}\right)\right\}_{n \geqq 0} \leqq K\left(F_{\mu+\partial \mu}\right)$. 
Proof. Let $\delta_{\mu}>0$ be given with $\mu+\delta_{\mu}<4$. Write $s=\left(\frac{1}{2} \delta_{\mu}-2 \delta_{f}\right) /\left(\mu+\delta_{\mu}-2\right)$. Define $g:[s, 1-s] \rightarrow[s, 1-s]$ by $g(x)=\left[\left(\mu+\delta_{\mu}\right) /(1-2 s)\right] \cdot(x-s)(1-s-x)+s$. This map $g$, which is topologically conjugate with $F_{\mu+\delta \mu}$ (namely $g=\Phi \circ F_{\mu+\delta \mu} \circ \Phi^{-1}$ with diffeo $\Phi:[0,1] \rightarrow[s, 1-s]$ given by $\Phi(x)=(1-2 s) x+s)$ has the properties: $g(s)=g(1-s)=s, g\left(\frac{1}{2}\right)=\frac{1}{4} \mu+\delta_{f}=F_{\mu}\left(\frac{1}{2}\right)+\delta_{f}$. For $\delta_{f}$ sufficiently small, we have $F_{\mu}\left(F_{\mu}\left(\frac{1}{2}\right)+\delta_{f}\right)-\delta_{f}>g\left(F_{\mu}\left(\frac{1}{2}\right)+\delta_{f}\right)$ and $F_{\mu}\left(F_{\mu}\left(F_{\mu}\left(\frac{1}{2}\right)+\delta_{f}\right)-\delta_{f}\right)-\delta_{f}>g\left(F_{\mu}\left(F_{\mu}\left(\frac{1}{2}\right)+\right.\right.$ $\left.\left.\delta_{f}\right)-\delta_{f}\right)$. Notice for $\delta_{f}=0$ the difference $\left[F_{\mu}\left(F_{\mu}\left(\frac{1}{2}\right)+\delta_{f}\right)-\delta_{f}\right]-g\left(F_{\mu}\left(\frac{1}{2}\right)+\delta_{f}\right)=$ $F_{\mu}\left(\frac{1}{4} \mu\right)-g\left(\frac{1}{4} \mu\right)$ only depends on the given $\mu$ and $\delta_{\mu}$.

Since the lengths of the noisy cylinders go to zero as $\delta_{f}$ goes to zero (this follows from the fact that the pre-images of the critical point under $F_{\mu}$ are dense in the interval, see Guckenheimer [13]), we obtain, in a similar manner as for the tent map, there exists noise level $\delta_{f}>0$ and a sequence $\left\{A_{n}\right\}_{0 \leqq n \leqq N}$ consisting of the symbols $L$ and $R$ except the last one which is equal to $C$, i.e., $A_{N}=C$ such that inf $J\left\{A_{0} \ldots A_{N} \mid\left(F_{\mu}, \delta_{f}\right)\right\}>\frac{1}{4} \mu+\delta_{f}$ and $\inf J\left\{A_{0} \ldots A_{N} \mid(g, 0)\right\}<\frac{1}{4} \mu+\delta_{f}$. Hence $\left\{A_{n}\right\}_{0 \leqq n \leqq N}<K(g)$.

Let $\left\{x_{n}\right\}_{n \geqq 0}$ be a non-critical $\delta_{f}$-pseudo $F_{\mu}$-orbit in $\operatorname{Inv}\left(F_{\mu}, \delta_{f}\right)$. We write $B_{n}=\operatorname{Symbol}\left(x_{n}\right)$ for $0 \leqq n \leqq N$. Since $\left\{x_{n}\right\}_{0 \leqq n \leqq N} \subset \operatorname{Inv}\left(F_{\mu}, \delta_{f}\right)$ we know $\inf J\left\{B_{0} \ldots B_{N} \mid\left(F_{\mu}, \delta_{f}\right)\right\}<\frac{1}{4} \mu+\delta_{f}$. Applying Prop. 2-3 (iii) gives $\left\{B_{n}\right\}_{0 \leqq n \leqq N}<$ $\left\{A_{n}\right\}_{0 \leqq n \leqq N}$. We obtain $\left\{B_{n}\right\}_{n \geqq 0}=\left\{\operatorname{Symbol}\left(x_{n}\right)\right\}_{n \geqq 0}<K(g)$. Thus $\left\{\operatorname{Symbol}\left(x_{n}\right)\right\}_{n \geqq 0}<$ $K\left(F_{\mu+\delta \mu}\right)$.

Proposition 4-9. For every $\delta_{x}>0$ there exist $\delta_{\mu}>0$ and $\delta_{f}>0$ such that for each non-critical $\delta_{f}$-pseudo $F_{\mu}$-orbit $\left\{x_{n}\right\}_{n \geq 0}$ we have: There exists a real $F_{\mu+\delta \mu}$-orbit $\left\{y_{n}\right\}_{n \geqq 0}$ so that $\left|x_{n}-y_{n}\right| \leqq \delta_{x}$ for each $n \geqq 0$.

Proof. Let $\delta_{x}>0$ be given. For each $\delta_{\mu}>0$ with $\mu+\delta_{\mu}<4$ we let $\delta_{f}>0$ be as in Prop. 4-8. For each non-critical $\delta_{f}$-pseudo $F_{\mu}$-orbit $\left\{x_{n}\right\}_{n \geqq 0}$ we write $\left\{y_{n}\right\}_{n \geqq 0}$ for a real $F_{\mu+\delta \mu}$-orbit whose itinerary equals that of $\left\{x_{n}\right\}_{n \geqq 0}$, i.e., $\operatorname{Symbol}\left(x_{n}\right)=$ $\operatorname{Symbol}\left(y_{n}\right)$ for each $n \geqq 0$. For a given $\delta_{f}$-pseudo $F_{\mu}$-orbit $\left\{x_{n}\right\}_{n \geqq 0}$ we write $A=\left\{A_{n}\right\}_{n \geqq 0}=\left\{\operatorname{Symbol}\left(x_{n}\right)\right\}_{n \geqq 0}$, and we define $\delta_{x}(A)=\sup \delta_{x}(A ; n)$ with $\delta_{x}(A ; n)=$ $\sup \left\{|x-y|: x\right.$ in $J\left\{A_{n} A_{n+1} \ldots \mid\left(F_{\mu}, \delta_{f}\right)\right\}, y$ in $\left.J\left\{A_{n} A_{n+1} \ldots \mid\left(F_{\mu}, \delta_{f}\right)\right\}\right\}$.

Let $\delta_{\mu}>0$ be so that all the $\delta_{x}(A)$ 's are less than $\delta_{x}$, i.e., $\sup \left\{\delta_{x}(A)\right.$ : A symbol sequence of non-critical $\delta_{f}$-pseudo $F_{\mu}$-orbit $\} \leqq \delta_{x}$.

Remark. Note that $\delta_{x}$ goes to zero as $\delta_{\mu}$ and $\delta_{f}$ go to zero.

4-E. Proof of the Results. Let $F_{\mu}: R \rightarrow R$ be defined by $F_{\mu}(x)=\mu x(1-x)$. We assume that $1<\mu<\mu+\delta_{\mu}<4$. For $\mu \geqq 3$ we recall that $\operatorname{Inv}\left(F_{\mu}, \delta_{f}\right)=\left[F_{\mu}\left(F_{\mu}\left(\frac{1}{2}\right)+\delta_{f}\right)-\right.$ $\left.\delta_{f}, F_{\mu}\left(\frac{1}{2}\right)+\delta_{f}\right]$ for $\delta_{f}$ sufficiently small.

Proof of Theorem B-1. Let $F_{\mu}$ be as in the theorem. For $1<\mu<3$ it is rather obvious, and therefore left to the reader. Assume from now on $3 \leqq \mu<4$. We consider two cases, namely (1) $F_{\mu}$ has an attractive periodic orbit, and (2) all the periodic points for $F_{\mu}$ are repelling. Case (1): By applying Prop. 4-5 and the existence Theorem E due to Milnor and Thurston, the desired result follows. Case (2): We obtain the result by using Prop. 4-8 and the existence Theorem E.

Proof of Theorem B-2. Let $F_{\mu}$ be as in the theorem. For $1<\mu<3$ it is left to the reader. Assume from now on that $3 \leqq \mu<4$. There are two cases, namley, (1) $F_{\mu}$ 
has an attractive periodic orbit, and (2) all the periodic points for $F_{\mu}$ are repelling. Case (1): From Prop. 4-6 it follows that $F_{\mu}$ permits same parameter shadowing. Further, the map $F_{\mu}$ also permits increased parameter shadowing because for $\delta_{\mu}>0$ sufficiently small we have that $F_{\mu+\delta \mu}$ has an attractive periodic orbit consisting of either $p$ or $2 p$ points which is contained in the noisy attractor $A_{p}\left(F_{\mu}, \delta_{f}\right)$. Case (2): Using Prop. 4-9 we obtain that $F_{\mu}$ permits increased parameter shadowing.

Proof of Theorem B-3. Let $F_{\mu}$ be as in the theorem and apply Prop. 4-7.

\section{Discussion About More General Results}

The tent map is a prototype for piecewise expanding unimodal maps. We have shown that this family permits increased parameter shadowing. This result can be generalized in several ways. Let $F$ be a continuous map from $[0,1]$ into itself such that: (i) $F(0)=F(1)=0$, (ii) there is $c$ such that $c \leqq F(c)<1, F^{\prime}(x) \geqq 1$ for $0 \leqq x<c$ and $F^{\prime}(x) \leqq-1$ for $c<x \leqq 1$. We consider the maps $f_{\mu}$ defined by $f_{\mu}(x)=\mu \cdot F(x)$ with $1<\mu<1 / F(c)$. We can show: If the topological entropy of $f_{\mu}$ is a strictly increasing function of the parameter $\mu$, then $f_{\mu}$ permits increased parameter shadowing. Furthermore, there are cases where it is not monotonic and so increased parameter shadowing will fail.

Also the second example we studied can be generalized. Let $F$ be a map from $[0,1]$ into itself so that (i) $F(0)=F(1)=0$, (ii) there is $c$ with $0<c \leqq F(c)<1$, $F^{\prime}(x)>0$ for $0 \leqq x<c$ and $F^{\prime}(x)<0$ for $c<x \leqq 1$, and (iii) $F$ has a negative Schwarzian derivative, i.e., $F^{\prime \prime \prime}(x) / F^{\prime}(x)-(3 / 2) \cdot\left[F^{\prime \prime}(x) / F^{\prime}(x)\right]^{2}<0$ for $x \neq c$. Consider the maps $f_{\mu}$ defined by $f_{\mu}(x)=\mu \cdot F(x)$ with $1<\mu<1 / F(c)$. We can prove: If the kneading sequence of $f_{\mu}$ is a non-decreasing function of $\mu$, then $f_{\mu}$ permits increased parameter shadowing except at saddle-node bifurcation values. However, the quadratic family is the only example we know having this property.

For maps with several critical points we can prove a result similar to Bowen's result concerning Axiom A diffeomorphisms. This differs from Bowen's result in that the map is not a diffeomorphism. From the definition and the results in $[28,29]$ we obtain: Let $f$ be an Axiom $\mathrm{A} \mathrm{C}^{2}$-map from a compact interval into itself. Then: for every $\delta_{x}>0$ there is $\delta_{f}>0$ such that each $\delta_{f}$-pseudo orbit of $f$ can be $\delta_{x}$-shadowed by a real orbit of $f$. The map $f_{\mu}$ in $\mathrm{Th}^{m}$. B-2 is an example of an Axiom A map whenever $f_{\mu}$ has an attractive periodic orbit.

Remark. It is very likely that Theorem B-3 can be improved in the following way: Assume that $F_{\mu}$ has a one-sided attractive periodic orbit. Then there exist $\delta_{x}{ }^{*}>0$ so that for each real number $\delta_{\mu}$ and every $\delta_{f}>0$ there is a $\delta_{f}$-pseudo $F_{\mu}$-orbit which cannot be $\delta_{x}^{*}$-shadowed by a real $F_{\alpha}$-orbit, with $\alpha=\mu+\delta_{\mu}$.

\section{References}

1. Alekseev, V. M., Jakobson, M. V.: Symbolic dynamics and hyperbolic dynamic systems. Phys. Rep. 75, 287-325 (1981)

2. Bowen, R.: On Axiom A diffeomorphisms. Reg. Conf. Ser. Math. 35, Providence, RI: Am. Math. Soc. 1978

3. Boyarsky, A.: Randomness implies order. J. Math. Anal. Appl. 76, 483-497 (1980)

4. Collet, P., Eckmann, J.-P.: Iterated maps on the interval as dynamical systems. Boston: Birkhäuser 1980 
5. Collet, P., Crutchfield, J. P., Eckmann, J.-P.: Computing the topological entropy of maps. Commun. Math. Phys. 88, 257-262 (1983)

6. Coven, E. M., Kan, I., Yorke, J. A.: Pseudo-orbit shadowing in the family of tent maps. Preprint

7. Crutchfield, J. P., Farmer, J. D., Huberman, B. A.: Fluctuations and simple chaotic dynamics. Phys. Rep. 92, 45-82 (1982)

8. Crutchfield, J. P., Packard, N. H.: Symbolic dynamics of noisy chaos. Physica 7D, 201-223 (1983)

9. Derrida, B., Gervois, A., Pomeau, Y.: Iterations of endomorphisms on the real axis and representation of numbers. Ann. Inst. Henri Poincare' 29, 305-356 (1978)

10. Derrida, B., Gervols, A., Pomeau, Y.: Universal metric properties of bifurcations of endomorphisms. J. Phys. A12, 269-296 (1979)

11. Douady, A., Hubbard, J. H.: Itérations des polynômes quadratiques complexes. C. R. Acad. Sci. Paris 294, 123-126 (1982)

12. Eckmann, J.-P.: Roads to turbulence in dissipative dynamical systems. Rev. Mod. Phys. 53, 643-654 (1981)

13. Guckenheimer, J.: Sensitive dependence to initial conditions for one dimensional maps. Commun. Math. Phys. 70, 133-160 (1979)

14. Guckenheimer, J.: Bifurcations of dynamical systems. CIME Lectures Bressanone 1978. Boston: Birkhäuser 1980

15. Guckenheimer J., Holmes, P.: Nonlinear oscillations, dynamical systems and bifurcation of vectorfields. Applied Mathematical Sciences Vol. 42. Berlin, Heidelberg, New York: Springer 1983

16. Jonker, L.: Periodic orbits and kneading invariants. Proc. Lond. Math. Soc. 39, 428-450 (1979)

17. Jonker, L., Rand, D.: Bifurcations in one dimension. I. Invent. Math. 62, 347-365 (1981) II. Invent. Math. 63, 1-15 (1981)

18. Lasota, A., Yorke, J. A.: On the existence of invariant measures for piecewise monotonic transformations. Trans. Am. Math. Soc. 186, 481-488 (1973)

19. Lasota, A., Yorke, J. A.: On the existence of invariant measures for transformations with strictly turbulent trajectories. Bull. Acad. Polon. Sci. Ser. Sci. Math. 25, 233-238 (1977)

20. Li, T.-Y., Yorke, J. A.: Period three implies chaos. Am. Math. Mon. 82, 985-992 (1975)

21. May, R. M.: Biological populations with nonoverlapping generations: Stable points, stable cycles and chaos. Science 186, 645-647 (1974)

22. May, R. M.: Simple mathematical models with very complicated dynamics. Nature 261, 459-467 (1976)

23. Milnor, J., Thurston, W.: On iterated maps of the interval I, II. Preprint Princeton 1977

24. Milnor, J.: The monotonicity theorem for real quadratic maps. Bonn: Mathematische Arbeitstagung Bonn 1983

25. Milnor, J.: On the concept of attractor. Commun. Math. Phys. 99, 177-195 (1985)

26. Misiurewicz, M.: Horseshoes for mappings of the interval. Bull. Acad. Polon. Sci. Ser., Sci. Math. 27, 167-169 (1979)

27. Misiurewicz, M., Szlenk, W.: Entropy of piecewise monotone mappings. Stud. Math. 67, 45-63(1980)

28. Nusse, H. E.: Chaos, yet no chance to get lost. Order and structure in the chaotic dynamical behaviour of one-dimensional noninvertible Axiom A mappings arising in discrete biological models. Thesis Rijksuniversiteit Utrecht 1983

29. Nusse, H. E. Qualitative analysis of the dynamics and stability properties for Axiom A maps. J. Math. Anal. Appl. (To appear)

30. Parry, W.: Symbolic dynamics and transformations of the unit interval. Trans. Am. Math. Soc. 122, 368-378 (1966)

31. Ruelle, D.: Small random perturbations of dynamical systems and the definition of attractors. Commun. Math. Phys. 82, 137-151 (1981)

32. Singer, D.: Stable orbits and bifurcations of maps of the interval. SIAM J. Appl. Math. 35, 260-267 (1978)

Communicated by J. N. Mather

Received October 29, 1986; in revised form July 14, 1987 
